This is the final peer-reviewed accepted manuscript of:

D. Fabiani, S. V. Suraci and S. Bulzaga

Aging Investigation of Low-Voltage Cable Insulation Used in Nuclear Power Plants

in 2018 IEEE Electrical Insulation Conference (EIC), San Antonio, TX, 2018, pp. 516-519

The final published version is available online at:

https://doi.org/10.1109/EIC.2018.8481139

Rights / License:

The terms and conditions for the reuse of this version of the manuscript are specified in the publishing policy. For all terms of use and more information see the publisher's website.

This item was downloaded from IRIS Università di Bologna (https://cris.unibo.it/)

When citing, please refer to the published version. 


\section{Aging investigation of low-voltage cable insulation used in nuclear power plants}

\author{
Davide Fabiani \\ Dept. of Electrical Electronic \& Inform. Eng. \\ University of Bologna \\ Bologna, Italy \\ davide.fabiani@unibo.it
}

\author{
Simone V. Suraci \\ Dept. of Electrical Electronic \& Inform. Eng. \\ University of Bologna \\ Bologna, Italy \\ simone.suraci@unibo.it
}

\author{
Stefano Bulzaga \\ Dept. of Electrical Electronic \& Inform. Eng. \\ University of Bologna \\ Bologna, Italy \\ stefano.bulzaga@studio.unibo.it
}

\begin{abstract}
This paper deals with the effect of a long storage time after thermal and radiation aging on electrical and mechanical properties of insulating materials used in nuclear power plant cables. Strong post-irradiation effects were found on XLPE cable insulation years after irradiation. In particular, a dramatic reduction of mechanical properties was observed, suggesting insulation crosslinking and/or oxidation, as well as an increase of the imaginary permittivity values, particularly in the low frequency range where interfacial phenomena are active. This latter could be associated likely to the increase of interface between amorphous and crystalline regions, thus supporting the evidence that post-irradiation effects may provide mostly polymer crosslinking during storage time at room temperature.
\end{abstract}

Keywords - XLPE, post-irradiation effect, permittivity, condition monitoring, electrical insulation, nuclear power plant

\section{INTRODUCTION}

Low-voltage electrical cables are extensively used throughout nuclear power plants (NPP) for power transmission, control of equipment and instrumentation, communication of signals and data. These cables can be exposed to a wide range of harsh environmental conditions and stresses, like e.g., radiation, temperature, mechanical stresses and humidity, particularly inside the containment area. Cables are often used to directly support the safe operation of the plant. Hence, early cable degradation could result in unavailability of important equipment for safety as well as causing dangerous plant transients and shutdowns. Since an accident at a NPP can be the cause of catastrophic effects, it is of utmost importance that all apparatus dedicated to the safe operation and shutdown of the plant continue to operate after a postulated accident event, even in aged conditions.

Most of the NPP commissioned in '70s and ' 80 s are now reaching end-of-life but decommission costs are pushing NPP regulator to ask for life extension of NPPs to 60 (possibly even 80) years [1]. Since electric cables are one of the long life equipment that have not considered for replacement during the design life of a NPP, usually 40 years, evaluating degradation condition and predicting residual life are very critical issues [2]. Finally, as new reactors are being constructed and many others are in planning for the next future, appropriate cable choice and use of effective condition monitoring (CM) techniques to assess cable condition from commissioning, can be very useful at a later time for an effective cable lifetime management.

Cable insulation and jackets are made of polymer-based compounds, whose degradation state is traditionally assessed through destructive methods, like tensile testing or thermal analysis [3]. Non-destructive techniques, based e.g. on electrical quantities are not commonly used in NPP to evaluate cable insulation condition. This was the aim of the European project FP7 ADVANCE (Aging Diagnostics and Prognostics of low voltage I\&C cables). The project, finished in 2014, was focused on the study and the analysis of the results of accelerated ageing tests performed on a representative selection of cables already installed in European NPPs in order to evaluate the ability of electrical CM techniques to detect local and global cable ageing. The results were compared and correlated to those obtained with more conventional CM techniques for validation. Electrical permittivity, for instance, was found to be effectively associated with oxidation induced by aging [4-6]. Unfortunately, Diffusion Limited Oxidation (DLO) sometimes complicates the scenario, causing inhomogeneous degradation of electrical insulation, which leads to unexpected behavior of electrical and physical properties of the material with aging time [7]. Moreover, postirradiation effects have been also observed which may change material properties several months or even years after irradiation, due to slow diffusion and reaction rate of some chemical radicals created by aging. Both phenomena have to be taken into account to develop effective condition monitoring techniques for electrical insulation under such stress conditions[8].

The aim of this paper is to investigate how electrical properties used for condition monitoring changes 4 years after irradiation aging has occurred on XLPE cable specimens which were kept in a storage environment at ambient air and room temperature.

\section{EXPERIMENTAL SETUP}

\section{A. Specimens}

I\&C Alcatel RG59B cable specimens with XLPE insulation for nuclear power plants are studied in this paper [4]. As known, low-voltage power and control cables of NPPs includes 
shielding components and an outer sheath other than primary insulation. In order to focus the study on the primary insulation, outer sheath and the electromagnetic shield were removed from cables.

Sample cables have been aged through radiation and temperature for different time periods and then analyzed at delivery time and after four years during which they were stored in uncontrolled air conditions, in order to underline the possible post-irradiation effect.

Cables analyzed in this paper are characterized by [4]:

- Conductor: copper, $\varnothing 0.6 \mathrm{~mm}$

- Electrical insulation: XLPE, thickness $1.6 \mathrm{~mm}$

- Dose rate: $0.42 \mathrm{kGy} / \mathrm{h}$

- Temperature: $85^{\circ} \mathrm{C}$

Nevertheless, forcing high dose rates, chemical reactions are limited by the time it takes for oxygen to diffuse into the material; that is why it may occur that aging treatment affects particularly the superficial layer of specimens. This phenomenon, mentioned in the previous section is known in literature as DLO and affected the specimens investigated in this paper due to the large thickness of cable insulation.

\section{B. Accelerated aging}

The accelerated ageing procedure was fulfilled at the ROZA facility (UJV Řež, Czech Republic). The ageing process has been performed through a ${ }^{60} \mathrm{Co} \gamma$-ray source with temperature set at $85^{\circ} \mathrm{C}$. The maximum ageing time was 1000 $\mathrm{h}$ and specimens were removed for analysis every 200h [4].

\section{Measurements}

Dielectric response of specimens was analyzed in the range of $10^{-2}-10^{6} \mathrm{~Hz}$ through a NovoControl Alpha Dielectric Analyzer V2.2 with applied voltage of $3 \mathrm{~V}_{\text {rms. Instrumentation }}$ setup applies input voltage to the central metal conductor, measuring the output signal from a metallic shield placed on the external part of the insulation.

Tensile testing was used to obtain information about the mechanical properties of a material, such as elongation-atbreak $(\mathrm{EaB})$, Young modulus and strength at break were measured by stretching the sample until it breaks while recording load and extension [9]. Tubular insulation samples were prepared removing the internal copper conductor and tests were carried out at room temperature. The instrumentation used was an Instron 5566 Universal Testing Machine, extension rate was set at $50 \mathrm{~mm} \mathrm{~min}^{-1}$. Due to results dispersion, from four to six tests have been carried out for each aging time.

\section{EXPERIMENTAL RESULTS}

\section{A. Dielectric spectroscopy results}

Dielectric spectroscopy results in terms of real $\left(\varepsilon^{\prime}\right)$ and imaginary $(\varepsilon$ '") part of permittivity as a function of frequency and for different aging times are reported in Figures 1 and 2, respectively. It can be noticed that, in most cases, both coefficients of permittivity increase with aging time.

The comparison between real part of permittivity at delivery time (Fig. 1.a) and after four years (Fig. 1.b) shows an interesting behavior. In the high frequency range, $\varepsilon$ ' decreases with storage time for all the aged samples. Moreover, the $\varepsilon$ ' variation range is smaller for the stored samples than for those tested after delivery. Almost the opposite can be seen at low frequencies, where the real part of permittivity rises during storage, keeping the same variation before and after storage. It is worth noting that a further polarization peak appears in the frequency range $10-100 \mathrm{~Hz}$ for the 4-years stored samples.
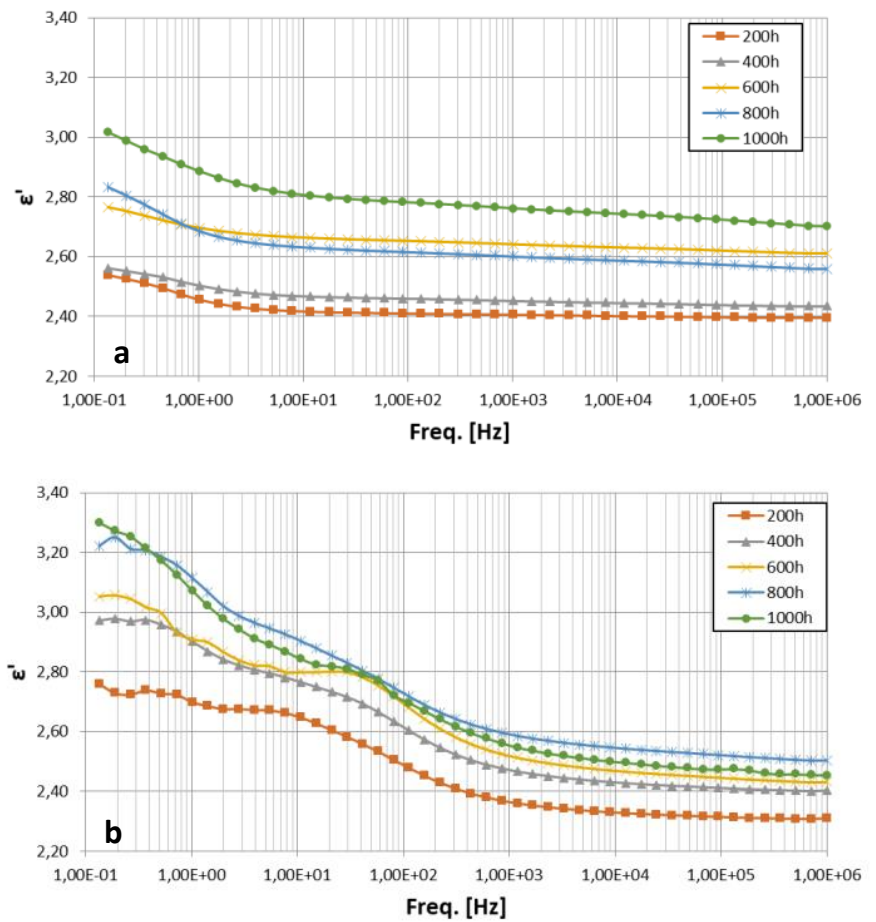

Fig. 1. Dielectric spectra as a fuction of frequency of real part of permittivity. Tests made at delivery time (a) and after four years of uncontrolled environment storage (b)

Figure 2 shows the trend of the imaginary part of permittivity at delivery time (Fig. 2.a) and after four years (Fig. 2.b) as a function of frequency. Imaginary permittivity appears to be more sensitive to aging, that is why it has been used as a marker to quantify condition of dielectrics [5, 6]. It is worth noting that Figures 2.a and 2.b show a totally different trend. Referring to $200 \mathrm{~h}$-aged sample at high frequencies, $\varepsilon$ "' increases about three times, but the longer the aging time, the lower the raise of $\varepsilon$ '. Indeed, in the 1000 h-aged sample, $\varepsilon$ ' increases by $5 \%$ only.

Again, Fig. 2.b shows a peak in the range of $10-100 \mathrm{~Hz}$ that is totally absent in the tests made at delivery time, likely due to additive consumption.

At low frequencies, the trend is quite unchanged. These little differences can be attributed to the higher-frequency peak already discussed. 


\section{DISCUSSION}
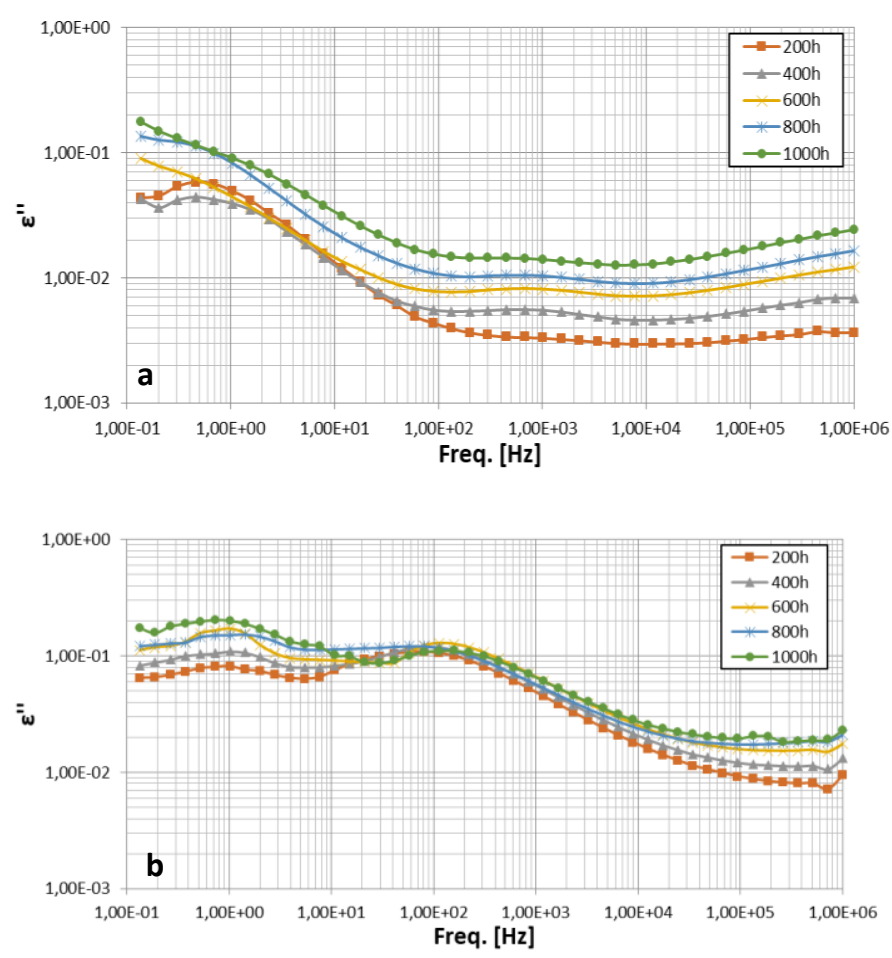

Fig. 2. Dielectric spectra as a fuction of frequency of immaginary part of permittivity. Tests made at delivery time (a) and after four years of uncontrolled environment storage (b).

\section{B. Mechanical results}

Mechanical results evidence even better the post irradiation effect, discussed in this paper.

Fig 3 shows the trend of $\mathrm{EaB}$ as a function of aging time. IEC Standards [9] set insulation failure at $50 \%$ of EaB. It can be noticed that, $1000 \mathrm{~h}$ irradiation time was needed to reach this limit for tests made at delivery time. On the contrary, after four-year storage $400 \mathrm{~h}$ of irradiation time are enough to reach the $50 \%$ end point. This behavior can be attributed to postirradiation effects that catalyze oxidation / crosslinking reactions even after irradiation source is turned off.

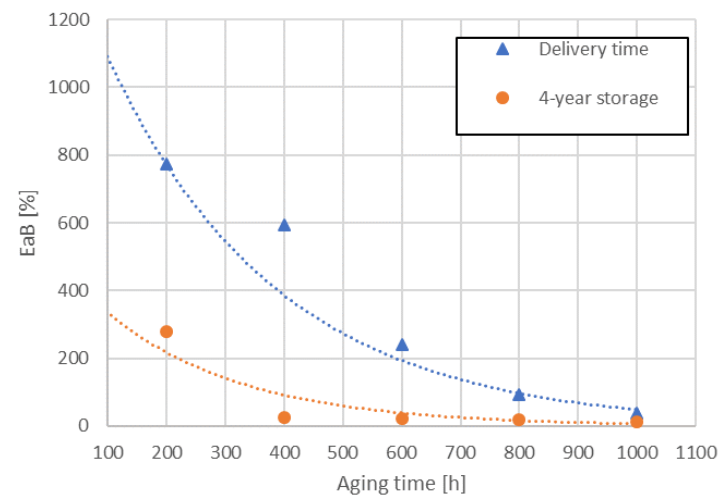

Fig. 3. Elongation at Break as a function of aging times. Interpolation made through an esponential tendency line.
As known in literature [5, 6] oxidation, with cross-linking and chain-scission, brings to the raise of losses that are reflected on the increase of $\varepsilon$ "' (see Fig. 2).

Post-irradiation effect can be explained through oxygen and radicals mobility inside different regions of polymers. Since diffusion constants of amorphous and crystalline region differs for orders of magnitudes of difference, oxygen propagation inside the crystalline part is very difficult. For this reason, oxidative reactions usually happen in the amorphous phase [8].

Radiation generates radicals inside both crystalline and amorphous parts of polymers. While radicals in amorphous region are able to bond each other or with oxygen, radicals created in the crystalline region remain trapped inside the matrix for some time after irradiation. These radicals slowly migrate to the interface between crystalline and amorphous region, where they meet molecules with oxygen and incept other oxidative reactions.

The presence of high dose rates prevents the diffusion of oxygen molecules in the inner part of specimens, so that oxygen groups can only be localized in the outer layers of the irradiated polymers, leading to DLO, as mentioned in the introduction [7].

Combined action of DLO and post-irradiation effect results in the different behavior of dielectric spectra at low and high frequencies both for real and immaginary part of permittivity discussed in the previous section.

Various authors attribute $\varepsilon$ " peaks at high frequencies to oxidation phenomena while low frequencies ones are usually a marker of additives consumption and structural modification (such as chain scission and crosslinking) [10]. The latter is usually imputed to interfacial modification between crystalline and amorphous regions of polymers, associated with MaxwellWagner-Sillars polarization [11].

The different response of dielectric spectra at low and high frequencies requires a quantity which could take into account the frequency range into which different modifications of polymers occur. This factor, called equivalent permittivity, is defined through the integral of permittivity at low and high frequency.

$$
\bar{\varepsilon}_{\text {high }}=\frac{\int_{f o}^{f_{\max }} \varepsilon d f}{d f} \quad \bar{\varepsilon}_{\text {low }}=\frac{\int_{f_{\min }}^{f_{o}} \varepsilon d f}{d f}
$$

where $\varepsilon$ is the complex permittivity, $f_{o}$ the delimitation frequency considered and $f_{\min , \max }$ is the minimum and maximum frequency of analyzed spectrum, respectively.

We focused our attention on imaginary part of permittivity because of the already discussed peculiarity and interesting trend of this parameter as a function of frequency and aging time [4].

Figure 4 shows imaginary part of equivalent permittivity $\left(\overline{\varepsilon "}_{\text {high }}\right.$ and $\overline{\varepsilon^{\prime \prime}}$ low) for both tests made at delivery time and after storage for different aging times. Note that a frequency of $10^{2} \mathrm{~Hz}$ has been used as delimitation marker for these dielectric responses. At frequency lower than $10^{2} \mathrm{~Hz}$, we usually have interfacial phenomena, while higher values are usually imputed to oxidation. 
It could be noticed that post-irradiation effect causes an increase of both parts of imaginary permittivity.

Referring to high frequencies equivalent imaginary permittivity (Fig. 4a) we can notice a raise of oxidation grade that increase linearly with aging time. This behavior can be explained through both the already discussed migration phenomena and radical production by continuous radiation.

The low-frequency part of equivalent imaginary permittivity shows a more important increase (up to 14 times) which can be correlated with $\mathrm{EaB}$ behavior (Fig. 3). The dramatic decrease of $\mathrm{EaB}$ after storage evidences that crosslinking of radical species can occur also years after irradiation. It is worth noting that $400 \mathrm{~h}$ of irradiation are able to reduce $\mathrm{EaB}$ from $600 \%$ to $30 \%$ in 4 years of storage, while further irradiation times do not significantly reduce the EaB. A similar behavior is shown for the equivalent permittivity, indicating thus a good correlation with crosslinking grade.

The plot of $\overline{\varepsilon^{\prime \prime}}$ high and $\overline{\varepsilon^{\prime \prime}}$ low underlines small differences between the two test periods, so that the results are not reported here for the sake of brevity.
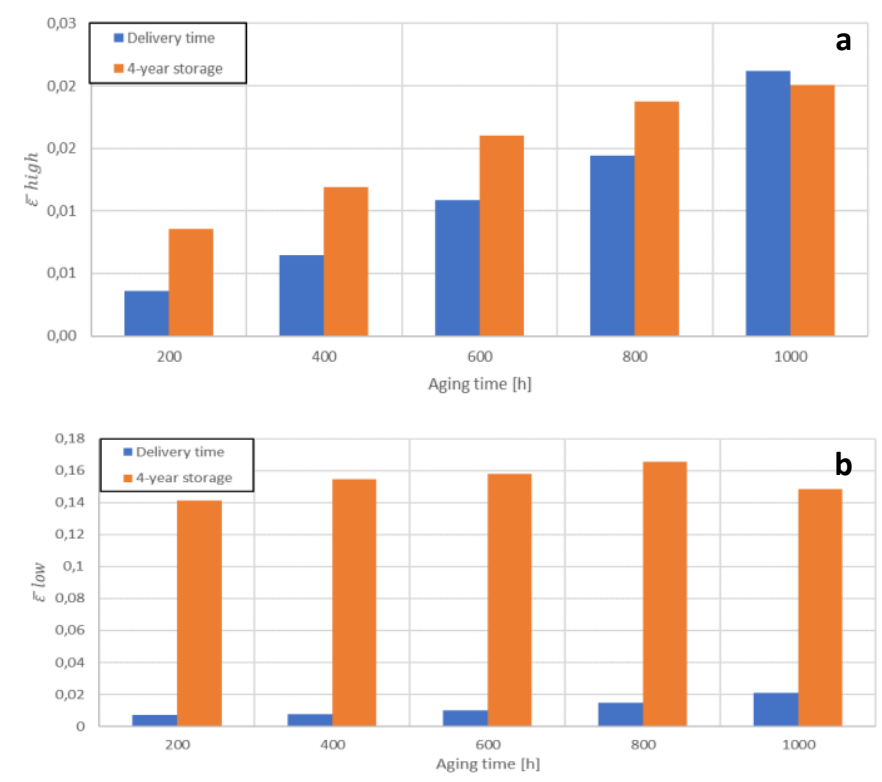

Fig. 4. High (a) and low (b) frequency equivalent immaginary permittivity trend as a function of aging time for the two tests periods.

\section{CONCLUSIONS}

Electrical and mechanical properties of the irradiated polymer here investigated show significant changes after years of storage under uncontrolled ambient conditions due to postirradiation effects. Experimental results show a good correlation between dielectric response and mechanical properties, before and after storage. Splitting dielectric spectrum in two parts allows crosslinking and oxidation effects to be singled out. In particular, the introduced equivalent permittivity can be a useful marker to quantify the aging degradation of insulating materials, underlying possible postirradiation effects. An important issue of this research is that once a polymer is irradiated with a high dose, like e.g. in case of a nuclear accident, insulation condition has not to be evaluated only immediately after accident. Under these circumstances, in fact, degradation evolves with time even in absence of any applied stress, therefore diagnostic measurements must be repeated with time, even years later.

\section{ACKNOWLEDGMENT}

The research has been developed thanks to the specimens provided within the FP7 EU project ADVANCE. The authors are grateful to Vit Placek of UJV Řež (Czech Republic) for specimen aging, Luca Verardi and Chiara Gualandi for helping with experiments.

\section{REFERENCES}

[1] International Atomic Energy Agency, "Management of life cycle and ageing at nuclear power plants - Improved I\&C maintenance", 2004.

[2] International Atomic Energy Agency, "Assessing and managing cable aging in nuclear power plants”, 2012.

[3] Sandia Laboratories, "Nuclear energy plant optimization (NEPO) - Final report on aging and condition monitoring of low-voltage cable materials", 2005.

[4] L. Verardi, "Aging of nuclear power plant cables: in search of nondestructive diagnostic quantities", $\mathrm{PhD}$ Thesis, University of Bologna, 2014.

[5] L. Verardi, D. Fabiani, G. C. Montanari, "Correlation of Electrical and Mechanical Properties in Accelerated Aging of LV Nuclear Power Plant Cables", IEEE ICHVE Poznan,Poland.

[6] E. Linde, L. Verardi, D. Fabiani, U.W. Gedde, "Dielectric spectroscopy as a condition monitoring technique for cable insulation based on crosslinked polyethylene", Polymer Testing.

[7] J. Wise, K.T. Gillen, R.L. Clough, Quantitative model for the time development of diffusion-limited oxidation profiles, Polymer, Volume 38, Issue 8, 1997, Pages 1929-1944.

[8] E. Suljovrujic, "Post-irradiation effects in polyethylenes irradiated under various atmospheres", Radiation Physics and Chemistry.

[9] "Nuclear power plants - Instrumentation and control important to safety - Electrical equipment condition monitoring methods Part 3: Elongation at break", IEC/IEEE 62582-3:2012 Standard

[10] S. Nakamura, F. Murabayashi, K. Iida, G. Sawa, and M. Ieda, "Degradation of dielectric properties of polyethylene by combined irradiation and thermal stresses", IEEE Transactions on Electrical Insulation, vol. 22, no. 6, pp. 715-720, 1987.

[11] M. Fu, G. Chen, L. A. Dissado, J. C. Fothergill and C. Zou, "The effect of gamma irradiation on space charge behaviour and dielectric spectroscopy of low-density polyethylene", IEEE Conference Winchester, UK. 